\title{
Fatty acid synthase expression in cutaneous melanocytic neoplasms
}

\author{
Payal Kapur ${ }^{1}$, Dinesh Rakheja ${ }^{1,2}$, Lonnie C Roy ${ }^{2}$ and Mai P Hoang ${ }^{1,2}$ \\ ${ }^{1}$ Department of Pathology, University of Texas Southwestern Medical Center, Dallas, TX, USA and \\ ${ }^{2}$ Children's Medical Center, Dallas, TX, USA
}

\begin{abstract}
Mammalian fatty acid synthase is a multifunctional enzyme complex involved in de novo synthesis of saturated fatty acids, and inhibitors of fatty acid synthase are being evaluated as potential therapeutic agents. Increased fatty acid synthase expression has been demonstrated in subsets of malignancies, including colon, breast, endometrium, prostate and ovarian carcinomas, and recently malignant melanomas. We evaluated the immunohistochemical expression of fatty acid synthase in 155 cutaneous melanocytic lesions. They included 30 congenital nevi, 19 compound nevi, 40 Spitz nevi, 48 primary melanomas, and 18 metastatic melanomas. Fatty acid synthase expression was stronger in malignant melanomas in comparison to conventional nevi and Spitz nevi, and was the highest for metastatic melanoma. Of the primary malignant melanomas, mean fatty acid synthase scores were significantly greater for Clark levels IV and V compared to Clark levels I and II $(P<0.001)$. In addition, melanomas with Breslow thickness $0.75-1.50 \mathrm{~mm}$ and $>1.50 \mathrm{~mm}$ showed significantly higher mean fatty acid synthase scores compared with those with Breslow thickness $<0.75 \mathrm{~mm}(P=0.013$ and $<0.001$, respectively). Of interest, congenital melanocytic nevi also showed strong fatty acid synthase expression, similar to that seen in metastatic melanoma. This may represent persistence of or regression to a fetal phenotype since normal fetal tissues are known to express high levels of fatty acid synthase.

Modern Pathology (2005) 18, 1107-1112. doi:10.1038/modpathol.3800395; published online 6 May 2005
\end{abstract}

Keywords: congenital melanocytic nevi; melanocytic nevi; malignant melanoma; Spitz nevi; fatty acid synthase; immunohistochemistry

Fatty acid synthase, the key enzyme responsible for the synthesis of fatty acids, catalyzes the conversion of acetyl-CoA and malonyl-CoA into long-chain fatty acids. ${ }^{1}$ It is weakly expressed in some normal adult human tissues. ${ }^{1}$ Increased fatty acid synthase expression has been demonstrated in subsets of malignancies, including colon, breast, and ovarian carcinomas. ${ }^{2-12}$ Recently, the expression of fatty acid synthase was shown to correlate with Breslow thickness in malignant melanoma while only weak expression was present in melanocytic nevi. ${ }^{13}$ In addition, the expression of fatty acid synthase may have therapeutic relevance because fatty acid synthase inhibitors, cerulenin and triclosan, have been demonstrated to be cytotoxic to human cancer cells in vitro. ${ }^{14,15}$ To date, there is no known

Correspondence: Dr MP Hoang, MD, Department of Pathology, University of Texas Southwestern Medical Center, 5323 Harry Hines Blvd, Dallas, TX 75390-9073, USA.

E-mail: mai.hoang@UTSouthwestern.edu

Abstract presented at the 94th Annual Meeting of the United States and Canadian Academy of Pathology, San Antonio, Texas, 2005.

Received 3 November 2004; revised 12 January 2005; accepted 13 January 2005; published online 6 May 2005 information on the staining pattern of fatty acid synthase in congenital melanocytic nevi; thus, the study of fatty acid synthase expression may shed light on their biology and possibly be of therapeutic value.

\section{Materials and methods}

The study has been approved by the internal review board at the University of Texas Southwestern Medical Center at Dallas. Archival materials of 19 compound melanocytic nevi, 40 Spitz nevi, 48 primary malignant melanomas (10 Clark level I, 10 Clark level II, nine Clark level III, 10 Clark level IV, nine Clark level V), 18 metastatic melanomas, and 30 congenital melanocytic nevi (Table 1), diagnosed between 1993 and 2003, were retrieved from the surgical pathology files of the University of Texas Southwestern Medical Center and Children's Medical Center at Dallas, Texas. Some of these cases have been previously published. ${ }^{16}$ Of the primary malignant melanomas, the Breslow depth was less than $0.75 \mathrm{~mm}$ in 25 cases, between 0.75 and $1.50 \mathrm{~mm}$ in seven cases, and greater than $1.50 \mathrm{~mm}$ in 16 cases. The following clinical information was obtained 
from the patients' medical records: age, sex, location of lesion, size of the lesion, duration, therapy, and follow-up.

\section{Immunohistochemistry}

One representative formalin-fixed and paraffinembedded block from each case was used to cut $4-\mu$ m-thick sections. The anti-fatty acid synthase antibody, a mouse monoclonal IgG (clone 14G5, Immuno-Biological Laboratories Co., Ltd, Japan), was used at concentration of $3 \mu \mathrm{g} / \mathrm{ml}$. The staining was performed manually by the standard peroxidase method, in which the secondary antibody (Dako, Carpinteria, CA, USA) was tagged with horseradish peroxidase and the chromogen was diaminobenzidine. The endogenous peroxidase activity was quenched by hydrogen peroxide. Nonspecific antibody binding was blocked with protein blocker (Dako). The sections were lightly counterstained by the Giemsa method so that unstained nuclei were light blue in color and any melanin pigment appeared green. ${ }^{17}$ The staining method was first standardized on sections of breast and colonic carcinomas. During standardization, steam-based antigen retrieval was attempted. However, the antibody was found to work better without the antigen retrieval; therefore, this step was not performed on the studied cases. The fatty acid synthase expression in sebaceous glands and the stratum granulosum of the epidermis was used as internal positive control, in accordance with the recent work of Uchiyama et $a .^{18}$ Appropriate negative control was included with each run.

Each case was scored separately by two of the authors in a blinded manner (PK and MPH). The cases, for which there was disagreement, were rescored jointly for consensus (PK, DR, and MPH).

Table 1 Demographic data of 155 melanocytic lesions

\begin{tabular}{lrrc}
\hline Tumor type & $\mathrm{N}$ & $\begin{array}{c}\text { Age range } \\
\text { (mean) (years) }\end{array}$ & Male:female \\
\hline Conventional nevi & 19 & $28-63(43)$ & $4: 11$ \\
Spitz nevi & 40 & $2-53(18)$ & $1: 1$ \\
Congenital nevi & 30 & 1 month-36 (7) & $2: 1$ \\
Primary melanoma & 48 & $35-97(67)$ & $1: 11$ \\
Metastatic melanoma & 18 & $42-92(65)$ & $6: 11$ \\
\hline
\end{tabular}

The staining intensity was graded subjectively from 0 to $3+$ (negative: 0 , low intensity: 1 , moderate: 2 , and strong: 3 ). The fraction of positive cells was estimated using a three-tiered scale $(<10 \%=0,10-$ $50 \%=1,51-80 \%=2,>80 \%=3)$. We used a combined scoring system, modified from that described by Innocenzi et al. ${ }^{13}$ The overall score was calculated as the average of both the intensity and the positive fraction score in each case.

\section{Statistical Analysis}

Descriptive analyses are presented with means, standard deviations, and error bar graphs. Statistical testing for differences in the percentages of positive immunostaining among groups was performed with analysis of variance and Bonferroni corrections for multiple comparisons. Two-tailed $P$-values of less than 0.05 were considered to be statistically significant.

\section{Results}

The demographic data of these 155 melanocytic lesions are summarized in Table 1. Of the congenital melanocytic nevi, one patient had neurofibromatosis. The maximum dimensions of the congenital melanocytic nevi ranged from 1.2 to $17 \mathrm{~cm}$ (mean: $5.6 \mathrm{~cm}$ ). The anatomic locations of the tumors were: upper extremity (5), lower extremity (4), head and neck (9), trunk (11), and not specified (1). All the patients are alive and without evidence of either recurrence or malignant transformation, with a follow-up ranging from 4 to 15 years (mean: 9.2 years). Of these 30 congenital nevi cases, 23 were histologically intradermal and seven were compound type.

Mean fatty acid synthase scores in conventional nevi, congenital nevi, Spitz nevi, malignant melanomas, and metastatic melanomas are presented in Table 2 and Figure 2. Positive fatty acid synthase staining was characterized by a cytoplasmic and granular pattern. The staining intensity was comparable to that of adjacent normal sebaceous glands (Figure 1d). The fatty acid synthase expression was the highest in metastatic melanomas and congenital melanocytic nevi, intermediate in primary malignant melanomas, and lowest in Spitz nevi and conventional nevi (Figure 2). Within the primary

Table 2 Multiple comparisons of fatty acid synthase expression in different tumor types with Bonferroni correction

\begin{tabular}{lccl}
\hline Tumor type & $\mathrm{N}$ & Mean \pm standard deviation & Significant difference between following group pairs (P<0.001) \\
\hline Conventional nevi (CN) & 19 & $0.21 \pm 0.42$ & CN:CgN, CN:M, CN:MetM \\
Spitz nevi (SN) & 40 & $0.5 \pm 0.72$ & SN:CgN, SN:M, SN:MetM \\
Congenital nevi (CgN) & 30 & $2.25 \pm 0.57$ & CgN:CN, CgN:SN, CgN:M \\
Primary melanoma (M) & 48 & $1.26 \pm 0.95$ & M:CN, M:SN, M:CgN, M:MetM \\
Metastatic melanoma (MetM) & 18 & $2.56 \pm 0.59$ & MetM:CN, MetM:SN, MetM:M
\end{tabular}



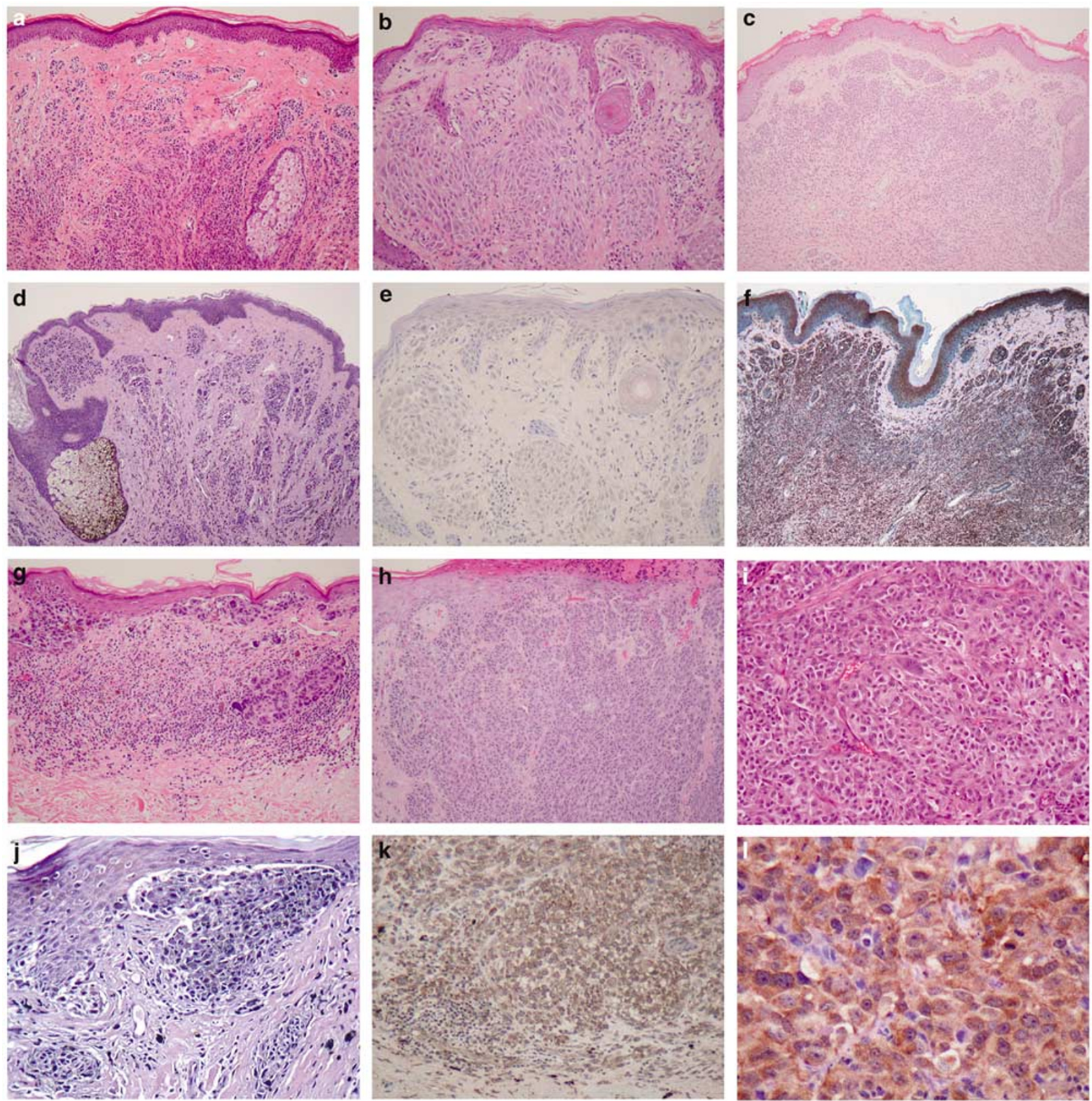

Figure 1 Histology and fatty acid synthase expression in (a, d) conventional nevus, (b, e) Spitz nevus, (c, f) congenital melanocytic nevus, (g, j) Clark level II malignant melanoma, (h, k) Clark level IV malignant melanoma, and (i, l) metastatic melanoma. Normal sebaceous gland demonstrates strong fatty acid synthase positivity (d). Fatty acid synthase expression is stronger in primary and metastatic melanomas $(\mathbf{j}, \mathbf{k}, \mathbf{l})$ in comparison to conventional (d) and Spitz (e) nevi. Of interest, strong fatty acid synthase expression is noted in both congenital melanocytic nevus (f) and metastatic melanoma (l).

malignant melanomas, fatty acid synthase score was significantly greater for Clark levels IV and V, when compared to Clark levels I and II (Figure 3). In addition, melanomas with Breslow thickness of $0.75-1.50 \mathrm{~mm}$ and greater than $1.50 \mathrm{~mm}$ showed a significantly higher fatty acid synthase score when compared with melanomas with Breslow thickness less than $0.75 \mathrm{~mm}$ (Figure 4).

\section{Discussion}

Mammalian fatty acid synthase (EC 2.3.1.85) is a multifunctional enzyme complex involved in de novo synthesis of saturated fatty acids from acetylCoA, malonyl-CoA, and NADPH. It is a homodimer with a molecular weight of $544 \mathrm{kDa}$. The gene for fatty acid synthase resides on chromosome $17 \mathrm{q} 25 .{ }^{19}$ 


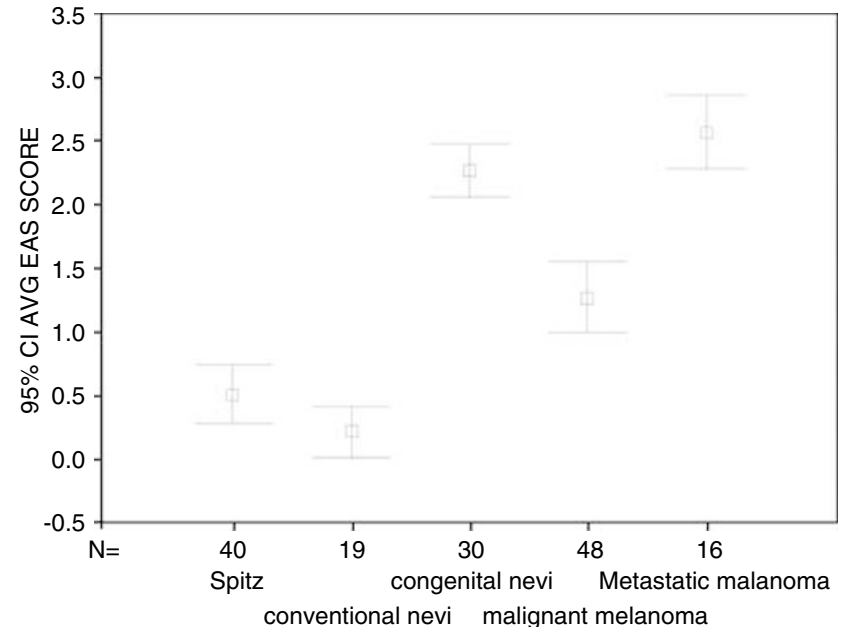

Figure 2 Mean and 95\% confidence interval of fatty acid synthase score of different tumor types.

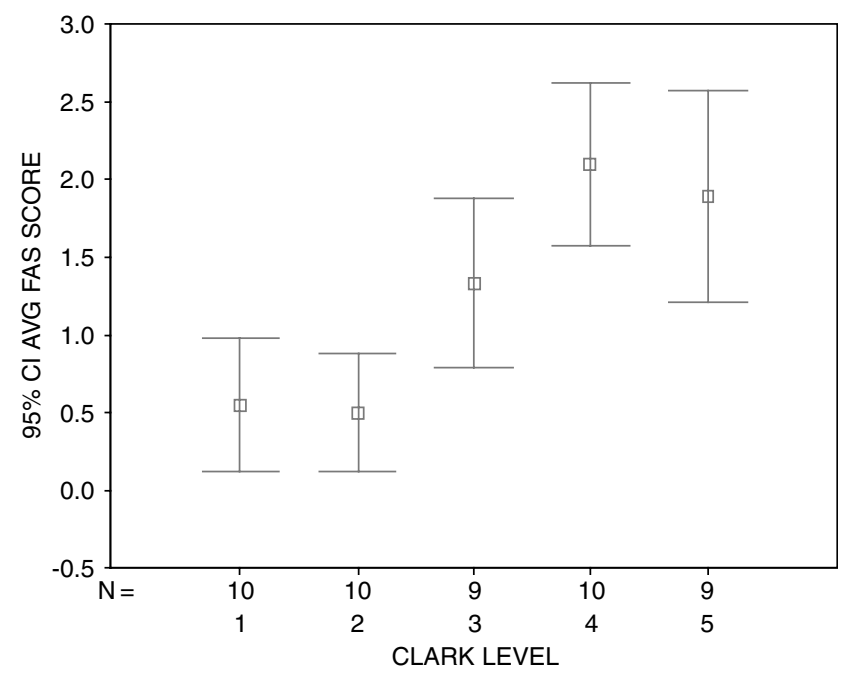

Figure 3 Mean and 95\% confidence interval of fatty acid synthase score of primary melanomas within different Clark levels. $P$-value is statistically significant $(0.001$ or $<0.001)$ for Clark level I:IV, I:V, II:IV, and II:V.

Kusukabe et $a l^{1}$ demonstrated the immunohistochemical expression of fatty acid synthase in various human adult and fetal tissues. Under normal conditions cells generally use circulating fatty acids from dietary lipids. Fatty acid synthase is overexpressed in neoplastic tisues because of their increased energy need. A high level of expression of fatty acid synthase has been demonstrated in aggressive subsets of human malignancies, including those of mammary (2), prostatic (3), colonic (4), ovarian (5), gastric (6), esophageal (7), pulmonary (8), oral (9), thyroid (10), mesothelial (11), endometrial (12), and melanocytic (13) origin. This information may possibly have therapeutic relevance

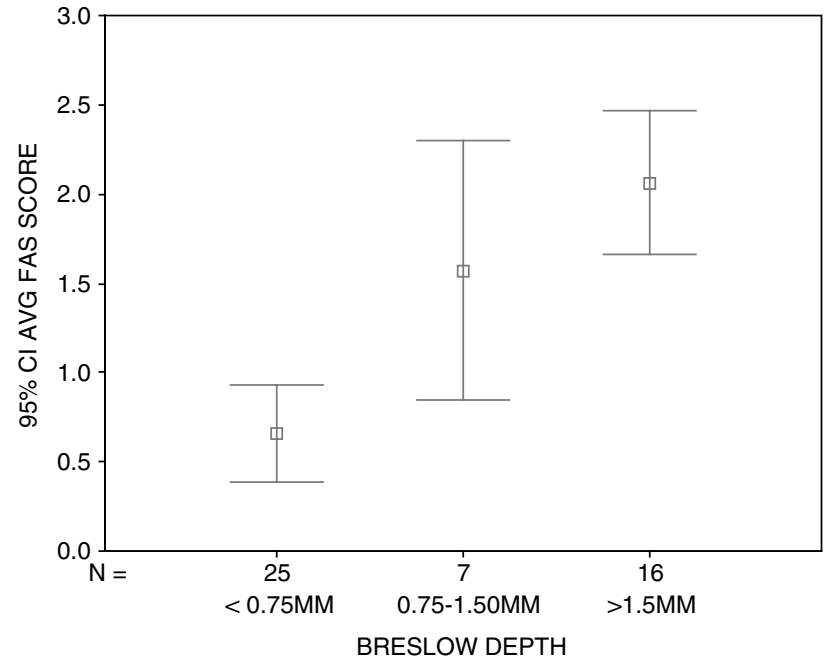

Figure 4 Mean and 95\% confidence interval of fatty acid synthase score of primary melanomas within different Breslow depth categories. $P$-value is less than 0.001 for Breslow depth $<0.75 \mathrm{~mm}$ : $>1.50 \mathrm{~mm}$. $P$-value is 0.013 for Breslow depth $<0.75 \mathrm{~mm}: 0.75-1.50 \mathrm{~mm}$.

because fatty acid synthase inhibitors, cerulenin and triclosan, have been shown to be cytotoxic to human cancer cells in vitro. ${ }^{14,15}$ Cerulenin, a natural antibiotic product of the fungus Cephalosporium cerulens, is a specific noncompetitive inhibitor of the beta-ketoacyl synthase activity of fatty acid synthase. ${ }^{14}$ In various cancer cell lines, it has been shown to be selectively cytotoxic to cancer cells that have increased fatty acid synthase, but not to normal cells that have low levels of fatty acid synthase. ${ }^{14,15}$

We observed increasing fatty acid synthase expression with increasing depth of invasion in malignant melanomas, with the highest fatty acid synthase expression seen in metastatic melanomas and low fatty acid synthase expression in conventional and Spitz nevi. Similarly, Innocenzi et $a l^{13}$ demonstrated statistical association of fatty acid synthase expression with Breslow thickness and overall survival in patients with malignant melanoma. Melanomas with thickness greater than $2 \mathrm{~mm}$ and strong fatty acid synthase expression were more likely to be lethal than those with weaker fatty acid synthase immunoreactivity. ${ }^{13}$ All of their metastatic melanoma cases showed high fatty acid synthase expression. ${ }^{13}$ In this study by Innocenzi et al, ${ }^{13}$ all 30 conventional melanocytic nevi showed only weak fatty acid synthase expression. Of note although mean fatty acid synthase expression was significantly different between Spitz nevi and primary melanomas in our study, overlap in distributions was observed between thin malignant melanomas and Spitz nevi.

The mechanism responsible for regulation of fatty acid synthase expression in cancer is not known at this time. Moreover, the regulation of fatty acid 
synthase in normal tissue is not fully understood. There is speculation that fatty acid synthase may play a role in tumor cell proliferation. Pizer et $a l^{20}$ found that in human colon cancer cell lines fatty acid synthase inhibition resulted in blockage of DNA replication and S-phase progression. In hormonal-sensitive tumors, estrogen, progesterone, and androgen have been demonstrated to play a role in fatty acid synthase regulation. ${ }^{21}$ Thus there appears to be multiple signaling pathways responsible for fatty acid synthase dysregulation in cancer. Additional studies are needed in order to define the role of fatty acid synthase in melanoma development, growth, and invasion.

We have shown that there is high level of fatty acid synthase expression in all 30 congenital nevi and the expression is comparable to that seen in metastatic melanomas. This overlap in immunoprofile is interesting. Congenital nevi are cutaneous melanocytic lesions that occur in approximately $1 \%$ of newborns. ${ }^{22,23}$ They are classified into three groups based on size: small (less than $1.5 \mathrm{~cm}$ ), medium $(1.5-19.9 \mathrm{~cm})$, and large or giant $(20 \mathrm{~cm}$ or greater). ${ }^{24}$ Large congenital melanocytic nevi have an increased risk for the development of cutaneous and extracutaneous melanomas. ${ }^{24-26}$ The overall incidence of malignant transformation of congenital melanocytic nevi ranges from 0.025 to $5.9 \%$ in different studies $(2-42 \%$ in large congenital nevi) with very high risk during the first decade of life. ${ }^{25,26}$ The expression of fatty acid synthase in congenital nevi could represent either persistence of or regression to a fetal immunophenotype, since it is known that fetal cells express high levels of fatty acid synthase. $^{1}$ The strong expression of fatty acid synthase in congenital nevi is not diagnostically helpful in the setting of malignant melanoma arising in congenital nevi. However, it raises the possibility of potential therapeutic use of fatty acid synthase inhibitors in the treatment of large and unresectable congenital nevi.

In summary, our findings show that melanomas express high levels of fatty acid synthase. The fatty acid synthase expression in melanomas increases with increasing depth of invasion, and is the highest for metastatic melanomas. We also demonstrate that conventional and Spitz nevi show a much lower expression of fatty acid synthase, consistent with their benign biologic behavior. Surprisingly, congenital nevi show very high levels of fatty acid synthase expression, near to that seen for metastatic melanomas.

\section{References}

1 Kusakabe T, Maeda M, Hoshi N, et al. Fatty acid synthase is expressed mainly in adult hormonesensitive cells or cells with high lipid metabolism and in proliferating fetal cells. J Histochem Cytochem 2000;48:613-622.
2 Alo PL, Visca P, Trombetta G, et al. Fatty acid synthase (FAS) predictive strength in poorly differentiated early breast carcinomas. Tumori 1999;85:35-40.

3 Shurbaji MS, Kalbfleisch JH, Thurmond TS. Immunohistochemical detection of a fatty acid synthase (OA519) as a predictor of progression of prostate cancer. Hum Pathol 1996;27:917-921.

4 Rashid A, Pizer ES, Moga M, et al. Elevated expression of fatty acid synthase and fatty acid synthetic activity in colorectal neoplasia. Am J Pathol 1997;150:201-208.

5 Gansler TS, Hardman III W, Hunt DA, et al. Increased expression of fatty acid synthase (OA-519) in ovarian neoplasms predicts shorter survival. Hum Pathol 1997;28:686-692.

6 Kusakabe T, Nashimoto A, Honma K, et al. Fatty acid synthase is highly expressed in carcinoma, adenoma and in regenerative epithelium and intestinal metaplasia of the stomach. Histopathology 2002;40:71-79.

7 Nemoto T, Terashima S, Kogure M, et al. Overexpression of fatty acid synthase in oesophageal squamous cell dysplasia and carcinoma. Pathobiology 2001;69: 297-303.

8 Piyathilake CJ, Frost AR, Manne U, et al. The expression of fatty acid synthase (FASE) is an early event in the development and progression of squamous cell carcinoma of the lung. Hum Pathol 2000;31: 1068-1073.

9 Krontiras H, Roye GD, Beenken SE, et al. Fatty acid synthase expression is increased in neoplastic lesions of the oral tongue. Head Neck 1999;21:325-329.

10 Sekiguchi M, Shiroko Y, Arai T, et al. Biological characteristics and chemosensitivity profile of four human anaplastic thyroid carcinoma cell lines. Biomed Pharmacother 2001;55:466-474.

11 Gabrielson EW, Pinn ML, Testa JR, et al. Increased fatty acid synthase is a therapeutic target in mesothelioma. Clin Cancer Res 2001;7:153-157.

12 Pizer ES, Lax SF, Kuhajda FP, et al. Fatty acid synthase expression in endometrial carcinoma: correlation with cell proliferation and hormone receptors. Cancer 1998; 83:528-537.

13 Innocenzi D, Alo PL, Balzani A, et al. Fatty acid synthase expression in melanoma. J Cutan Pathol 2003;30:23-28.

14 Kuhajda FP, Jenner K, Wood FD, et al. Fatty acid synthesis: a potential selective target for antineoplastic therapy. Proc Natl Acad Sci USA 1994;91:6379-6383.

15 Liu B, Wang Y, Fillgrove KL, et al. Triclosan inhibits enol-reductase of type I fatty acid synthase in vitro and is cytotoxic to MCF-7 and SKBr-3 breast cancer cells. Cancer Chemother Pharmacol 2002;49:187-193.

16 Kapur P, Selim MA, Roy LC, et al. Spitz nevi and atypical Spitz nevi/tumors: a histologic and immunohistochemical analysis. Mod Pathol 2004;18:197-204.

17 Kamino H, Tam ST. Immunoperoxidase technique modified by counterstain with azure B as a diagnostic aid in evaluating heavily pigmented melanocytic neoplasms. J Cutan Pathol 1991;18:436-439.

18 Uchiyama N, Yamamoto A, Kameda K, et al. The activity of fatty acid synthase of epidermal keratinocytes is regulated in the lower stratum spinulosum and the stratum basale by local inflammation rather than by circulating hormones. J Dermatol Sci 2000;24:134-141.

19 Jayakumar A, Chirala SS, Chinault AC, et al. Isolation and chromosomal mapping of genomic clones encoding the human fatty acid synthase gene. Genomics 1994;23:420-424. 
20 Pizer ES, Chrest FJ, DiGiuseppe JA, et al. Pharmacological inhibitors of mammalian fatty acid synthase suppress DNA replication and induce apoptosis in tumor cell lines. Cancer Res 1998;58:46114615.

21 Kuhajda FP. Fatty acid synthase and human cancer: new perspectives on its role in tumor biology. Nutrition 2000;16:202-208.

22 Alper JC, Holmes LB. The incidence and significance of birthmarks in a cohort of 4,641 newborns. Pediatr Dermatol 1983;1:58-68.
23 Walton RG, Jacobs AH, Cox AJ. Pigmented lesions in newborn infants. Br J Dermatol 1976;95:389-396.

24 Kopf AW, Bart RS, Hennessey P. Congenital nevocytic nevi and malignant melanomas. J Am Acad Dermatol 1979;1:123-130.

25 Berg P, Lindelof B. Congenital melanocytic nevi and cutaneous melanoma. Melanoma Res 2003;13:441-445.

26 Bittencourt FV, Marghoob AA, Kopf AW, et al. Large congenital melanocytic nevi and the risk for development of malignant melanoma and neurocutaneous melanocytosis. Pediatrics 2000;106:736-741. 